\title{
PREMATURITY IN PREGNANCIES OF MOTHERS WITH SYSTEMIC LUPUS ERYTHEMATOSUS IN A TERTIARY HOSPITAL
}

\author{
Sergio Henrique Oliveira dos Santos ${ }^{1, *}$, Juliana Bühring ${ }^{1}$, Bárbara Seabra Carneiro으. Domingos Sávio Nunes de Lima² \\ 1. Hospital Universitário Getúlio Vargas, Manaus (AM), Brazil. 2.Universidade Federal do Amazonas, Manaus (AM), Brazil. \\ ${ }^{*}$ Corresponding author: sergio.henrique@live.com
}

\section{BACKGROUND}

Systemic lupus erythematosus (SLE) is an autoimmune disease that has a variable clinical picture and a remitting-recurrent character. It can reach a female:male ratio 9:1, affecting negatively maternal reproductive aspect, as disease exacerbation occurs in about $50 \%$ of pregnancies. Preterm labor (PL) is defined as one between 20 and 37 weeks of gestation, the proportion of PL in Brazil between 2003 and 2018 was $8.8 \%$. The objectives of the present research were to describe the prevalence and incidence of disease activity (DA) of pregnancies of mothers diagnosed with SLE six month before pregnancy (6MBP), at moment pregnancy was noted in follow-up (MPN) and during pregnancy (DP), to compare the proportion of PL in the sample, and to verify whether there is correlation between disease activity, and prematurity.

\section{MATERIALS AND METHODS}

A descriptive cross-sectional study was performed in a rheumatology service of a tertiary hospital that follows about 800 patients with SLE, being approximately 700 female patients. The patients who had at least one pregnancy from January 2003 until December 2019, were in regular follow-up and had a born alive infant were included. Patients unable to provide data about the gestational age at labor were excluded; categorical variable was tested using the Fisher's exact test and the proportions were tested using the binomial test.

\section{RESULTS}

Eighty-two patients were included with 104 pregnancies; the mean age at pregnancy was 28.01 years, standard deviation (SD) 5.4 years and the mean disease length was 6.3 years SD 4.3 years. The mean pregnancy length was 36.8 weeks SD 3.06 weeks. Forty-one (39.4\%) had disease activity 6MBP, $32(30.7 \%)$ at MPN and $56(53.8 \%)$ in any moment DP. From 63 patients were not active before pregnancy, eight (12.6\%) became active at MPN and 23 (36.5\%) in any moment during pregnancy (Fig. 1). There was prematurity in 34 pregnancies (32.7\%). The proportion was significantly higher than general population (32.7 vs. 8.8\%, $p<0.001$, $\mathrm{OR}=5.04,95 \% \mathrm{Cl}=3.9-6.5)$ (Fig. 2). There was association between disease activity $6 \mathrm{MBP}$ and prematurity $(\mathrm{p}=0.021, \mathrm{OR}=2.59,95 \%$ $\mathrm{Cl}=1.11-6)$; at $\mathrm{MPN}(\mathrm{p}=0.003, \mathrm{OR}=3.67,95 \% \mathrm{Cl}=1.518-8.857)$; and in any moment $\mathrm{DP}(\mathrm{p}=0.004, \mathrm{OR}=3.49,95 \% \mathrm{Cl}=1.43-8.56)$.

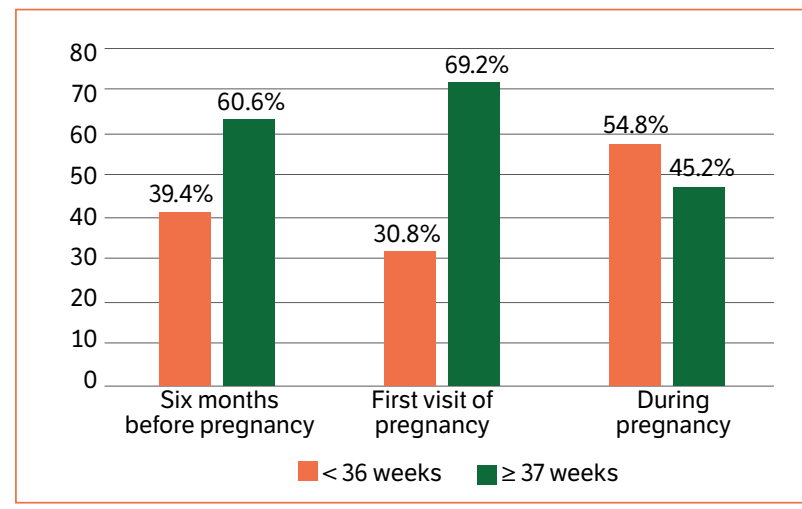

Figure 1. Distribution of frequency of disease activity by period related to pregnancy. 


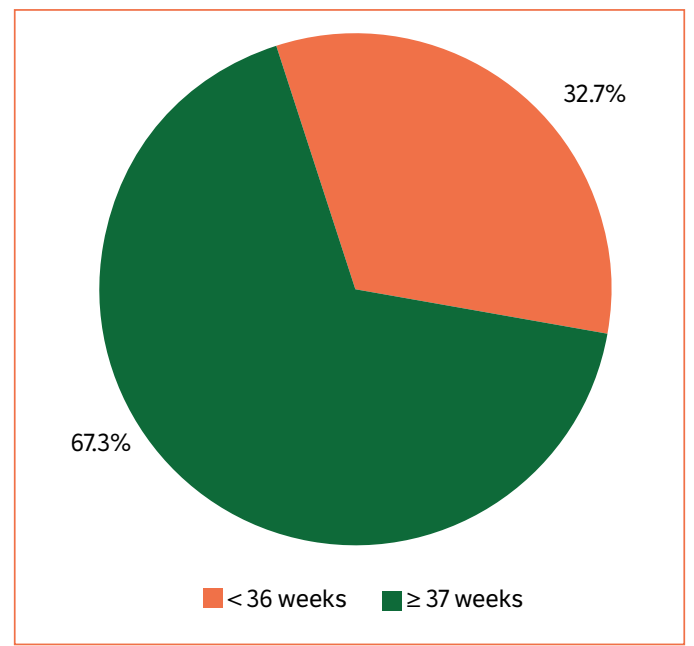

Figure 2. Frequency of preterm labor in 104 pregnancies from 82 patients.

\section{CONCLUSION}

These results allowed us to conclude that there was a higher proportion of prematurity in our sample than in general population and disease activity six months before, at moment pregnancy was noted in follow-up, as well as activity during pregnancy were risk factors to prematurity. 